\title{
Article \\ Phenotypic and Molecular Characteristics of the MDR Efflux Pump Gene-Carrying Stenotrophomonas maltophilia Strains Isolated in Warsaw, Poland
}

\author{
Olga M. Zając ${ }^{1}\left(\mathbb{D}\right.$, Stefan Tyski $^{1,2}\left(\mathbb{D}\right.$ and Agnieszka E. Laudy ${ }^{1, *(\mathbb{C}}$ \\ 1 Department of Pharmaceutical Microbiology, Medical University of Warsaw, 02091 Warsaw, Poland; \\ olga.zajac@wum.edu.pl (O.M.Z.); s.tyski@nil.gov.pl (S.T.) \\ 2 Department of Antibiotics and Microbiology, National Medicines Institute, 02091 Warsaw, Poland \\ * Correspondence: alaudy@wp.pl
}

check for updates

Citation: Zając, O.M.; Tyski, S.; Laudy, A.E. Phenotypic and Molecular Characteristics of the MDR Efflux Pump Gene-Carrying Stenotrophomonas maltophilia Strains Isolated in Warsaw, Poland. Biology 2022, 11, 105. https://doi.org/ 10.3390/biology11010105

Academic Editor: Vincent Sanchis-Borja

Received: 10 December 2021

Accepted: 5 January 2022

Published: 10 January 2022

Publisher's Note: MDPI stays neutral with regard to jurisdictional claims in published maps and institutional affiliations.

Copyright: (C) 2022 by the authors. Licensee MDPI, Basel, Switzerland. This article is an open access article distributed under the terms and conditions of the Creative Commons Attribution (CC BY) license (https:// creativecommons.org/licenses/by/ $4.0 /)$
Simple Summary: Nosocomial infections caused by Stenotrophomonas maltophilia have been increasing worldwide. These bacteria are intrinsically resistant to most antibiotics. The underestimated resistance mechanism of Gram-negative rods is an overexpression of multidrug-resistant (MDR) efflux pumps. The aim of this study was to analyze the genetic diversity of isolates derived from various clinical materials, including blood, and the prevalence of MDR efflux pump genes and susceptibility profiles to the anti-S. maltophilia drugs. The research was conducted on 94 S. maltophilia isolates derived from hospitalized patients and outpatients in Warsaw, Poland. All isolates were susceptible to trimethoprim-sulfamethoxazole and minocycline, while 44/94 isolates demonstrated reduction in susceptibility to levofloxacin. A large genetic variation was observed among these isolates. However, a clonal relationship was revealed among two groups of bloodstream isolates from one hospital ward: (1) nine isolates, (2) six isolates. Moreover, the presence of genes encoding ten different efflux pumps from the resistance-nodulation-division family and the ATP-binding cassette family was shown in the majority of the 94 isolates. The obtained knowledge about the prevalence of efflux pump genes in clinical S. maltophilia strains makes it possible to predict the scale of the risk of resistance emergence in strains as a result of gene overexpression.

Abstract: An increase of nosocomial infections caused by Stenotrophomonas maltophilia strains has recently been observed all over the world. The isolation of these bacteria from the blood is of particular concern. In this study we performed the phenotypic and genotypic characterization of 94 S. maltophilia isolates, including isolates from patients hospitalized in a tertiary Warsaw hospital $(n=79)$ and from outpatients $(n=15)$. All isolates were found to be susceptible to trimethoprimsulfamethoxazole and minocycline, while 44/94 isolates demonstrated a reduction in susceptibility to levofloxacin. A large genetic variation was observed among the isolates tested by pulsed-field gel electrophoresis. A clonal relationship with $100 \%$ similarity was observed between isolates within two sub-pulsotypes: the first included nine bloodstream isolates and the second involved six. Multilocus sequence typing showed two new sequence types (ST498 and ST499) deposited in public databases for molecular typing. Moreover, the presence of genes encoding ten different efflux pumps from the resistance-nodulation-division family and the ATP-binding cassette family was shown in the majority of the 94 isolates. The obtained knowledge about the prevalence of efflux pump genes in clinical S. maltophilia strains makes it possible to predict the scale of the risk of resistance emergence in strains as a result of gene overexpression.

Keywords: antibiotic susceptibility; efflux systems; molecular typing; MLST; PFGE; non-fermentative rods

\section{Introduction}

Stenotrophomonas maltophilia is one of the most frequently isolated non-fermentative Gram-negative bacteria responsible for nosocomial infections [1-3]. This opportunistic 
pathogen is dangerous for patients with coexisting diseases like acquired immunodeficiency syndrome (AIDS), cystic fibrosis, or cancer (particularly lung cancer), as well as patients with other immunodeficiencies, immunosuppressive therapy, mechanical ventilation, catheters, and those hospitalized for long periods. The critically ill patients from intensive care units, especially after broad-spectrum antibiotic therapy, are most exposed to infections. S. maltophilia strains mostly cause nosocomial respiratory tract infections (pneumonia), which can be associated with bloodstream infections. These bacteria can cause many other severe infections, including respiratory infections associated with acute exacerbations of chronic obstructive pulmonary disease, eye infections (endophthalmitis, keratitis, scleritis), skin and soft tissue infections, meningitis, endocarditis, and biliary sepsis. Infections caused by S. maltophilia are characterized by a high mortality rate, up to $69 \%$ in patients with bacteremia [3]. Although it is mainly a nosocomial pathogen, communityacquired infections are increasingly observed. Infections caused by this bacterium occur in both adults and children [1,2]. Infection occurs most often through direct contact with the aerosol created by a person with pneumonia. Other possible routes of transmission involve the hands of healthcare workers, dental unit suction tubing, contaminated endoscopes, or tap water. S. maltophilia strains, like most non-fermentative Gram-negative bacteria, are common in wet or moist environments (e.g., in taps or washbasin siphons and their surroundings) [2].

S. maltophilia infections are difficult to treat because they are intrinsically resistant to a majority of antibiotics and chemotherapeutic agents. According to the Clinical and Laboratory Standards Institute (CLSI), recommendations for Group A antimicrobial agents which are appropriate for use in the routine treatment of $S$. maltophilia infection includes only the three following antibiotics: trimethoprim-sulfamethoxazole, levofloxacin, and minocycline. Several mechanisms contribute to $S$. maltophilia resistance, among them: $\beta$-lactamases production, the activity of multidrug-resistant (MDR) efflux pumps, the excretion of other enzymes modifying antibiotic structures, and the production of proteins that protect the drug targets [2,4].

One underestimated resistance mechanism of Gram-negative rods, including S. maltophilia strains, is an overexpression of MDR efflux pumps. Efflux pumps described so far in Gram-negative bacteria can be classified into five families, called superfamilies, as follows: the ABC (ATP-binding cassette) family, the RND (resistance-nodulation-division) family, the MFS (major facilitator superfamily), the SMR (small multidrug resistance) family, and the MATE (multidrug and toxic compound extrusion) family. Of these families, mainly RND efflux systems contribute to antimicrobial resistance. RND efflux systems are able to remove antimicrobial agents belonging to different classes of antibiotics, chemotherapeutic agents, and disinfectants from bacterial cells [4]. These systems are tripartite complexes consisting of inner membrane proteins (IMPs), outer membrane proteins (OMPs), and membrane fusion proteins (MFPs) located in the periplasm combining the IMP and the OMP subunits. These efflux pumps are activated by a proton motive force to extrude agents into the extracellular environment. The RND efflux systems are encoded by genes organized in operons located in bacterial chromosomes. Only two of all known efflux pumps of Gram-negative rods are coded by genes located in conjugational plasmids, that is, the OqxAB and QepA efflux pumps reported among Enterobacteriaceae strains.

In contrast to Enterobacteriaceae and Pseudomonas aeruginosa, the MDR efflux pumps have been relatively sparsely investigated in S. maltophilia strains. This applies to both their prevalence among clinical and environmental strains and their role in the resistance of these bacilli. Until recently, it was thought that 12 MDR efflux systems occur in S. maltophilia. These systems belong to three different families of MDR efflux pumps: the RND family (SmeDEF [5-7], SmeABC [8], SmeIJK [9], SmeYZ [9], SmeVWX [10], SmeOP [11], SmeMN [12], and SmeGH [12]); the ABC family (SmrA [13] and Ma-cABCsm [14]); the MFS family (EmrCABsm) [15]; and the FuaABC pump [16], which is not classified. Moreover, the identification of the next two new MDR efflux pumps from the ABC family, SmaCDEF and SmaAB, in S. maltophilia was published in 2021 [17]. There is currently ongoing research 
on the occurrence of these MDR efflux systems among S. maltophilia isolated from humans and animals.

Recent investigations have revealed the high genetic diversity among S. maltophilia strains isolated in different parts of the world [18,19]. Molecular methods are used to provide evidence of epidemiological relationships between isolates. These methods are also an important tool in the investigation of the spread of $S$. maltophilia infections all over the world. Rizek et al. sequenced and analyzed the whole genomes of four clinical isolates of S. maltophilia, and for the presence of genes encoding the efflux pump systems [20]. The following MDR efflux pump genes have been identified: $s m e A B C$ (in two out of four isolates), smeDEF (in three out of four isolates), smeZ (in three out of four), smrA (in one out of four), and $m a c B$ (in all isolates), among others. It is therefore possible that there are no genes encoding the different pumps in the tested isolates. On the other hand, the presence of efflux-system-coding operons is not synonymous with the resistance of such a strain to antibiotics that are substrates of the MDR pumps. Only the overexpression of efflux pump genes causes resistance or decreased susceptibility of a strain to antibiotics. So, if most clinical S. maltophilia strains possess the genes listed above, the scale of the existing danger of the emergence of resistance, even MDR, is huge.

The aim of this study was to analyze the genetic diversity of isolates derived from various clinical materials, including blood, and the prevalence of genes encoding MDR efflux pumps and susceptibility profiles to the main three anti-S. maltophilia drugs. The research was conducted on a collection of 94 S. maltophilia clinical isolates obtained from hospitalized patients and outpatients in Warsaw, Poland.

\section{Materials and Methods}

\subsection{Bacterial Strains}

The research was conducted on a collection of 94 non-duplicate $S$. maltophilia clinical isolates obtained from adult patients hospitalized in one tertiary hospital in Warsaw $(n=79)$ and from outpatients $(n=15)$. Isolates were derived between January 2010 and October 2013 from blood samples (27), bronchial secretions (17), anus swabs (14), wound swabs (8), urine samples (6), sputum (4), drain swabs (3), stoma swabs (2), oral cavity swabs (2), eye swabs (2), gastrostomy swabs (2), ear swabs (2), peritoneal fluid, a bile sample, fluid from the pleural cavity, a vagina swab, and a nose swab. Biochemical identification of $S$. maltophilia isolates was performed by the Vitek-2 Compact system (bioMérieux, Mercy l'Etoile, France). All isolates were stored in Luria Bertani broth (BioMaxima SA, Lublin, Poland) with 20\% glycerol at $-80{ }^{\circ} \mathrm{C}$ until analysis. Two reference strains of $S$. maltophilia ATCC 13,637 and S. maltophilia ATCC 12,714 were also included in this study.

\subsection{Antimicrobial Susceptibility Testing}

The susceptibility of clinical isolates to levofloxacin, minocycline, and trimethoprimsulfamethoxazole was examined by the disc-diffusion test, according to the Clinical and Laboratory Standards Institute (CLSI) recommendations [21], and by the minimum inhibitory concentration (MIC) determination using Etests (Liofilchem srl, Roseto deli Abruzzi, Italy) [22]. Both assays were determined on Mueller-Hinton II agar medium (Becton, Dickinson and Company, Franklin Lakes, NJ, USA). The results of the susceptibility of the isolates were evaluated after incubation at $35^{\circ} \mathrm{C}$ for $18 \mathrm{~h}$ and interpreted according to the CLSI criteria [23]. Escherichia coli ATCC 25,922 was used as a reference strain for quality control in the antimicrobial susceptibility testing.

\subsection{Detection of MDR Efflux Pump Genes}

The molecular detection of genes encoding the MDR efflux pumps from the RND family (SmeDEF, SmeABC, SmeIJK, SmeYZ, SmeOP, SmeGH, SmeMN, SmeVWX) and the ABC family (SmrA, MacABCsm) was performed by polymerase chain reaction (PCR). The total DNA of the clinical isolates was extracted using a Genomic Mini Kit (A\&A Biotechnology, Gdynia, Poland). The PCR reactions were performed using Maxima Hot 
Start Taq DNA polymerase (Thermo Scientific, Thermo Fisher Scientific, Waltham, MA, USA) with the following amplification parameters: $95^{\circ} \mathrm{C}$ for $4 \mathrm{~min}$, followed by 25 cycles of $30 \mathrm{~s}$ at $95{ }^{\circ} \mathrm{C}, 30 \mathrm{~s}$ at $58{ }^{\circ} \mathrm{C}, 59{ }^{\circ} \mathrm{C}$ or $63{ }^{\circ} \mathrm{C}$ (annealing temperature for each pair of primers is described in Table 1), $60 \mathrm{~s}$ at $72{ }^{\circ} \mathrm{C}$, and a final extension for $5 \mathrm{~min}$ at $72{ }^{\circ} \mathrm{C}$. The sequences of the primers were derived from references or designed for this project based on gene sequences available in GenBank National Center for Biotechnology Information (NCBI) (https:/ / www.ncbi.nlm.nih.gov/, accessed on 1 February 2018). Primers used in this study are listed in Table 1. The reference S. maltophilia ATCC 13,637 strain harboring the MDR efflux pump genes was used. By sequencing the PCR reaction products obtained for the reference strain with primers listed in Table 1, the correct use of these primers for the detection of the specific pumps encoding genes was confirmed.

Table 1. Primers for the amplification of genes encoding MDR efflux pumps.

\begin{tabular}{|c|c|c|c|c|c|c|}
\hline $\begin{array}{c}\text { Efflux } \\
\text { System }\end{array}$ & $\begin{array}{l}\text { Target } \\
\text { Gene }\end{array}$ & Primer & Sequence $\left(5^{\prime}-3^{\prime}\right)$ & $\begin{array}{c}\text { Product } \\
\text { Length (bp) }\end{array}$ & $\begin{array}{c}\text { Annealing } \\
\text { Temperature } \\
\left({ }^{\circ} \mathrm{C}\right)\end{array}$ & Reference \\
\hline \multirow{2}{*}{ SmeABC } & $s m e B$ & $\begin{array}{l}\text { B-F } \\
\text { B-R }\end{array}$ & $\begin{array}{c}\text { GGGCCGGAAAGCTACGA } \\
\text { AGCGAAATGGTCACGAATGG }\end{array}$ & 200 & 59 & Chang et al. [8] \\
\hline & smeA & $A-F$ & AAGGCCATCGATGGCAAGGC & 146 & 59 & \multirow{2}{*}{ This study } \\
\hline \multirow{4}{*}{ SmeDEF } & \multirow{4}{*}{ smeD } & & 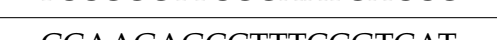 & \multirow[b]{2}{*}{150} & & \\
\hline & & $\begin{array}{l}\mathrm{D}-\mathrm{H} \\
\mathrm{D}-\mathrm{R}\end{array}$ & $\begin{array}{l}\text { CCAAGAGCCTTTCCGTCAT } \\
\text { TCTCGGACTTCAGCGTGAC }\end{array}$ & & 59 & Zhang et al. [6] \\
\hline & & RT-D-F & CGGTCAGCATCCTGATGGA & \multirow{2}{*}{73} & \multirow{2}{*}{59} & \\
\hline & & RT-D-R & ACGCTGACTTCGGAGAACTC & & & Leon et al. [7] \\
\hline \multirow{2}{*}{ SmeYZ } & \multirow{2}{*}{ smeZ } & Z-F & AGTGGACCAGCCAGTCGCT & \multirow{2}{*}{508} & \multirow{2}{*}{59} & \multirow{2}{*}{ This study } \\
\hline & & Z-R & ACTACATAGAAGACCGGCACG & & & \\
\hline \multirow{4}{*}{ SmeIJK } & \multirow{2}{*}{ smeK } & $\mathrm{K}-\mathrm{F}$ & GACCTCGCAGACGCAGTCG & \multirow{2}{*}{505} & \multirow{2}{*}{59} & \multirow{2}{*}{$\begin{array}{l}\text { Gould et al. [9] } \\
\text { modified }\end{array}$} \\
\hline & & $\mathrm{K}-\mathrm{R}$ & CAGGTAGTCGCGCAGGGTC & & & \\
\hline & \multirow{2}{*}{ smeI } & $\mathrm{I}-\mathrm{F}$ & TTCCGCGAAGGCCAGGAAGT & \multirow{2}{*}{107} & \multirow{2}{*}{59} & \multirow{2}{*}{ This study } \\
\hline & & $\mathrm{I}-\mathrm{R}$ & TCGTTCTGGCGCTTGGCTG & & & \\
\hline \multirow{2}{*}{ SmeOP } & \multirow{2}{*}{ smeP } & P-F & GGTGCTGGCGATGACCTTC & \multirow[b]{2}{*}{372} & & This study \\
\hline & & P-R & TCCGGCAGCA TCTTGTCGC & & 58 & \\
\hline & & N-F & GGTCTCCTCG ACCATGGAC & & & This study \\
\hline SmeMN & $\operatorname{smeN}$ & N-R & CCTTGCCCAGCGGGATG & 314 & 58 & inis study \\
\hline & & W-F & TTCGGCGACATCGTGCTCAA & & & \\
\hline SmeVWX & smeW & W-R & CTTGAAGAAGCGGTTGAACGG & 843 & 58 & Ihis study \\
\hline & & $\mathrm{H}-\mathrm{F}$ & GTGGATGATCGGCTTCACGAT & 556 & 58 & This study \\
\hline smeGH & smeH & $\mathrm{H}-\mathrm{R}$ & CGCATAGCCCTGGTCTTCTT & 506 & 58 & InIs stuay \\
\hline & & MacB-F & GTGATCGACGAGAACACCCA & & & Th \\
\hline MacABLsm & maсь & MacB-R & GGCCGATCATCGAGCCCA & 589 & 58 & 1111 \\
\hline & $s m r$ & SmrA-F & GGTGTGGCCGGTGCTGCT & 67. & 63 & Th \\
\hline SmrA & smrA & SmrA-R & CGCGGTGCTTGACCGCCA & 6/7 & 63 & IIIIs stuay \\
\hline
\end{tabular}

$\mathrm{F}$, forward primer; $\mathrm{R}$, reverse primer.

\subsection{Pulsed-Field Gel Electrophoresis (PFGE)}

All 94 S. maltophilia isolates were typed by PFGE according to a protocol published by Jumaa et al. [24] with modifications. An overnight culture of bacteria with a density of about $10^{8} \mathrm{CFU} / \mathrm{mL}$ was suspended in $150 \mu \mathrm{L}$ of cell suspension buffer and mixed with $20 \mu \mathrm{L}$ of $20 \mathrm{mg} / \mathrm{mL}$ Proteinase K (Promega GmbH, Walldorf, Germany) and $170 \mu \mathrm{L}$ of $1.5 \%$ low-melting agarose (SeaKem Gold Agarose Lonza, Basel, Switzerland) to form a plug with bacteria cells. The total DNA in agarose plugs was obtained by material lysis in $2.5 \mathrm{~mL}$ lysis buffer supplemented with $20 \mu \mathrm{L}$ of $20 \mathrm{mg} / \mathrm{mL}$ Proteinase K (Promega GmbH, Walldorf, Germany) and $7.5 \mu \mathrm{L}$ of $10 \mathrm{mg} / \mathrm{mL}$ RNase (Sigma, St. Louis, MO, USA) for $2.5 \mathrm{~h}$ at $55^{\circ} \mathrm{C}$. Plugs were washed and digested with $15 \mathrm{U}$ of $\mathrm{XbaI}$ restriction enzyme (Thermo 
Scientific, Thermo Fisher Scientific, Waltham, MA, USA) for $3 \mathrm{~h}$ at $37^{\circ} \mathrm{C}$. Electrophoresis was performed on the CHEF DR II Variable Angle System (Bio-Rad, Hercules, CA, USA). The electrophoresis conditions were as follows: total run time $20 \mathrm{~h}$ (the first-block switch time was 1 to $12 \mathrm{~s}$ for $13 \mathrm{~h}$, and the second-block switch time was 5 to $35 \mathrm{~s}$ for $7 \mathrm{~h}$ ), voltage $6 \mathrm{~V} / \mathrm{cm}$, switch angle $120^{\circ}$, temperature $14^{\circ} \mathrm{C}$. The total DNA of Salmonella serotype Braenderup strain (H9812) digested with XbaI enzyme (ABO, Gdańsk, Poland) was used as the DNA molecular-weight marker. The obtained PFGE profiles were analyzed using GelCompare II software (Applied Maths, Sint-Martens-Latem, Belgium), using the Dice coefficient and clustering by UPGMA with $1 \%$ tolerance. According to Tenover et al. [25] the isolates were clustered in the PFGE pulsotypes (PTs). Isolates with banding pattern similarity over $80 \%$ were considered to be related.

\subsection{Multilocus Sequence Typing (MLST)}

The total DNA of selected clinical isolates was extracted using a Genomic Mini Kit (A\&A Biotechnology, Gdynia, Poland). The methodology of MLST typing was carried out according to the Kaiser et al. [26] protocol. The sequences of the following seven highly conserved housekeeping genes were analyzed: atpD, gapA, guaA, mutM, $n u o D, p p s A$, and recA. The sequences of these primers are included in S. maltophilia MLST database (https: / / pubmlst.org/organisms/stenotrophomonas-maltophilia/primers, accessed on 1 February 2020). Sequencing of the obtained DNA templates was carried out in The Laboratory of DNA Sequencing and Oligonucleotides Synthesis, Institute of Biochemistry and Biophysics, Polish Academy of Science in Warsaw, Poland. The received DNA sequences were analyzed using Vector NTI Advance 11 software (Invitrogen, Thermo Fisher Scientific, Waltham, MA, USA) and compared with S. maltophilia MLST database (https:/ / pubmlst.org/organisms/stenotrophomonas-maltophilia/, accessed on 1 February 2020). A combination of the allelic sequences of all seven genes enabled the definition of the sequence type (ST) for each isolate. The new allelic sequences of housekeeping genes and new STs were submitted to the S. maltophilia MLST database (https: / / pubmlst.org/organisms/stenotrophomonas-maltophilia/, accessed on 19 June 2020).

\section{Results}

\subsection{Susceptibility Profiles of the Isolates}

Table 2 shows the susceptibility patterns of $94 \mathrm{~S}$. maltophilia isolates for three antibacterial agents. All isolates were susceptible to minocycline and trimethoprim-sulfamethoxazole, regardless of the assay used. Moreover, a minority of the studied isolates were nonsusceptible to levofloxacin (44 isolates in the Etest method and 8 isolates in the disc-diffusion method). The highest level of resistance to levofloxacin (MIC $=16 \mathrm{mg} / \mathrm{L}$ ) was obtained only for isolates 9/2010,30/2011, and 41/2011. The remaining four out of seven levofloxacinresistant isolates showed an MIC value of $8 \mathrm{mg} / \mathrm{L}$.

Table 2. The drug susceptibility of $S$. maltophilia clinical isolates $(n=94)$.

\begin{tabular}{|c|c|c|c|c|c|c|c|c|c|c|}
\hline \multirow{3}{*}{ Agent } & \multicolumn{4}{|c|}{ Disc-Diffusion Method } & \multicolumn{6}{|c|}{ Etest Method } \\
\hline & \multicolumn{3}{|c|}{ No. of Isolates (\%) } & \multirow{2}{*}{$\begin{array}{c}\text { GIZ Range }{ }^{a} \\
(\mathrm{~mm})\end{array}$} & \multicolumn{3}{|c|}{ No. of Isolates (\%) } & \multirow{2}{*}{$\begin{array}{l}\text { MIC Range } \\
\text { (mg/L) }\end{array}$} & \multirow{2}{*}{$\begin{array}{l}\mathrm{MIC}_{50} \\
(\mathrm{mg} / \mathrm{L})\end{array}$} & \multirow{2}{*}{$\begin{array}{l}\mathrm{MIC}_{90} \\
(\mathrm{mg} / \mathrm{L})\end{array}$} \\
\hline & $\mathrm{S}$ & I & $\mathbf{R}$ & & $\mathrm{S}$ & I & $\mathbf{R}$ & & & \\
\hline Minocycline & $94(100)$ & 0 & 0 & $21-36$ & 94 (100) & 0 & 0 & $0.19-3$ & 0.75 & 1.5 \\
\hline Levofloxacin & $86(91)$ & $6(6)$ & $2(2)$ & $9-34$ & $50(53)$ & $37(39.5)$ & $7(7.5)$ & $1-16$ & 2 & 6 \\
\hline $\begin{array}{l}\text { Trimethoprim- } \\
\text { sulfamethoxazole }\end{array}$ & $94(100)$ & 0 & 0 & $18-37$ & $94(100)$ & 0 & 0 & $0.047-0.75$ & 0.125 & 0.25 \\
\hline
\end{tabular}

S, susceptible strain; I, intermediate strain; R, resistant strain; GIZ, growth inhibition zone of the strain; MIC, the minimum inhibitory concentration of agent. ${ }^{a}$ Range of obtained diameters of the bacteria growth inhibition zones. 


\subsection{Occurrence of Genes Encoding the MDR Efflux Systems}

The PCR analysis of the distribution of MDR efflux system genes among the tested isolates revealed the presence of the $s m e D, s m e N, s m e H$, and $m a c B$ genes in all isolates, and the following genes encoding other efflux pumps: smeW and smrA in 93 out of the 94 isolates, smeP in 90 isolates, and smeZ in 89 . However, when searching for the SmeIJK and SmeABC efflux systems, the smeK and smeB genes were detected in only 69 out of 94 studied isolates. In the case of isolates with a negative result, the smeI and smeA genes were amplified in the second stage. We found that 17 out of $25 \mathrm{smeK}$-negative isolates harbored the smeI gene. On the other hand, the smeA gene was only detected among 3 out of 25 smeB-negative isolates. The occurrence of MDR efflux systems in the studied isolates of S. maltophilia is shown in Table 3, and the presence of the particular genes encoding these systems is given in Table S1 (Supplementary Materials).

Table 3. The occurrence of MDR efflux systems in S. maltophilia clinical isolates $(n=94)$.

\begin{tabular}{ccc}
\hline Efflux System Family & Efflux System & No. of Isolates (\%) \\
\hline & SmeABC & $72(76.6)$ \\
& SmeDEF & $94(100)$ \\
SmeIJK & $86(91.5)$ \\
\multirow{2}{*}{ RND } & SmeYZ & $89(94.7)$ \\
& SmeMN & $94(100)$ \\
& SmeOP & $90(95.7)$ \\
& SmeVWX & $93(98.9)$ \\
& SmeGH & $94(100)$ \\
\hline \multirow{2}{*}{ ABC } & SmrA & $93(98.9)$ \\
& MacABCsm & $94(100)$ \\
\hline
\end{tabular}

Among the 94 collected clinical isolates of S. maltophilia, PCR analysis showed the presence of 10 different MDR efflux systems -8 from the RND family and 2 from the ABC family -in 63 isolates. Meanwhile, 31 isolates showed the lack of one (21 isolates) or two (10 isolates) MDR efflux systems. In most isolates lacking two efflux systems (i.e., 6 out of 10 isolates), no genes encoding the SmeABC and SmeIJK systems were detected. In the remaining three isolates, the SmeABC and SmeYZ systems were not revealed, and in one isolate the SmeABC and SmrA systems were not detected.

\subsection{Molecular Typing of Isolates by Pulsed-Field Gel Electrophoresis (PFGE)}

Genetic relatedness between the 94 studied clinical isolates of S. maltophilia was assessed using PFGE analysis (Figure 1). Isolates were considered as a cluster if the similarity was at least $80 \%$. The PFGE analysis of the obtained isolate band patterns revealed 11 clusters, named A-K, which contained at least two tested isolates. Only 41 out of the 94 studied isolates met these requirements and were mapped to clusters. The isolates included in the clusters were assigned the appropriate pulsotype number. The two groups of isolates were obtained among the largest A cluster (i.e., nine isolates created A1 PT and six isolates created A3 PT). Within each of these groups, the similarity of the isolates was $100 \%$. These isolates were obtained from blood samples collected between September 2010 and February 2013. Moreover, only three pairs of isolates with PTs (D1, H1, and I1) obtained 100\% similarity in the PFGE patterns. 


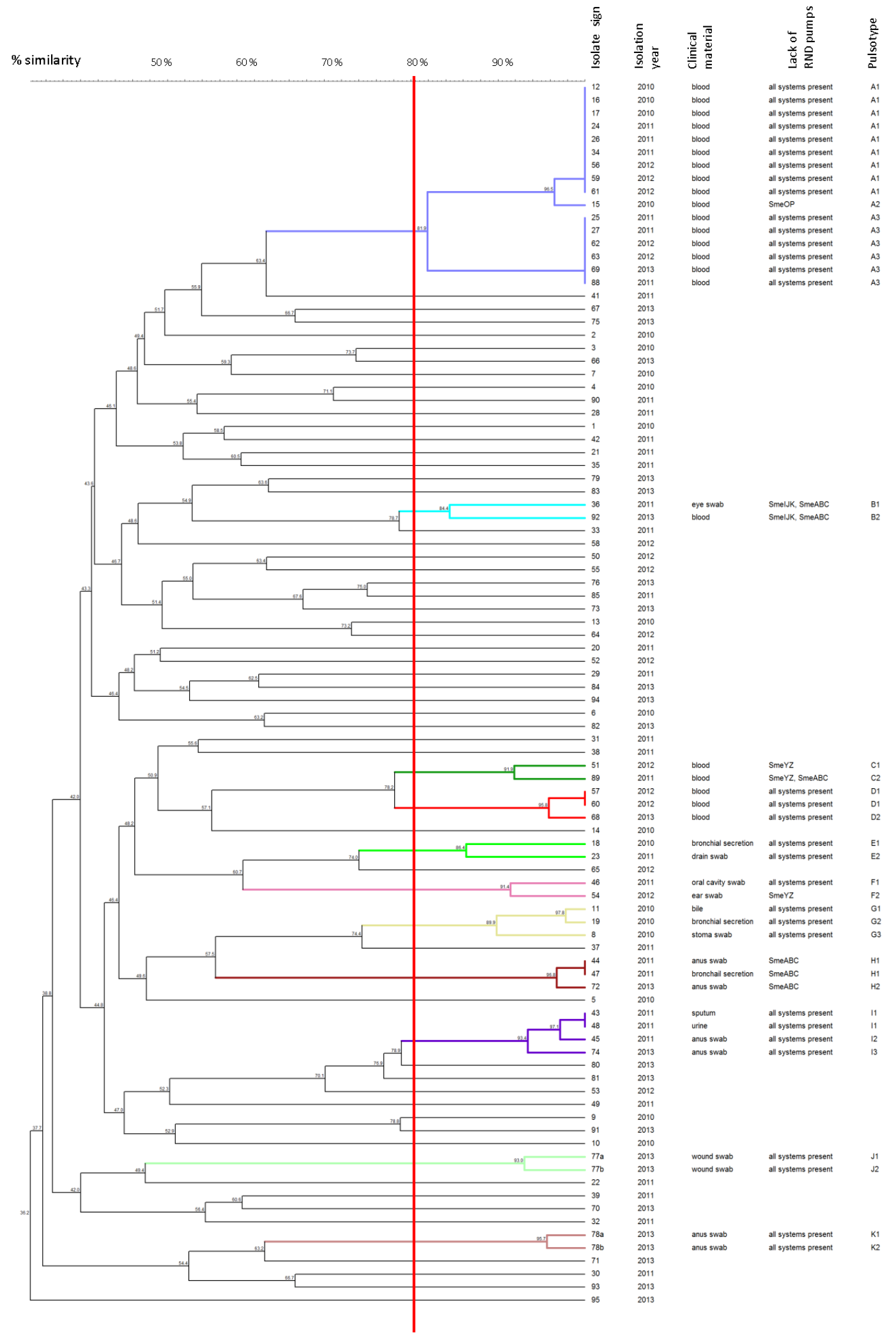

Figure 1. Analysis of PFGE patterns of the S. maltophilia isolates. The dendrogram presents the percentage similarity of PFGE profiles, the isolation year, the pulsotype mapped to clusters, and the following data about the named PTs: specified absence of the RND efflux systems and clinical material of isolation. The solid line indicates $80 \%$ similarity and is used to define the clusters with appropriate PTs. Isolates mapped to different clusters are marked with the various colors. In contrast, isolates not grouped in clusters are marked in black. 


\subsection{Multilocus Sequence Typing (MLST) Analysis}

The MLST analysis was performed on three selected S. maltophilia isolates from the most important clinical material (blood). The following representative isolates from clusters containing isolates with 100\% similarity were tested: no. 56/2012 with A1 PT (formed from nine blood isolates), no. 62/2012 with A3 PT (made up of six blood isolates), and no. 57/2012 with D1 PT (containing two blood isolates). The isolates mapped in these clusters showed the presence of all MDR efflux systems tested. Information regarding the MLST data of studied isolates is presented in Table 4. The MLST analysis showed that all three investigated isolates represent two novel MLST profiles, marked as sequence types-ST498 and ST499. Isolates 56/2012 from A1 PT and 62/2012 from A3 PT had an identical allele pattern, where five out of seven alleles of the highly conserved housekeeping genes analyzed revealed new sequences. Both isolates belong to the new group ST498. The third tested isolate, 57/2012 with D1 PT, also had new gene alleles and was classified into the second new MLST profile, ST499. All new sequences of alleles of conserved housekeeping genes and new ST profiles were deposited in public databases for molecular typing (https:/ / pubmlst.org/organisms/stenotrophomonas-maltophilia/, accessed on 19 June 2020).

Table 4. Alleles and sequence types in S. maltophilia isolates.

\begin{tabular}{|c|c|c|c|c|c|c|c|c|}
\hline \multirow{2}{*}{ Strain } & \multicolumn{7}{|c|}{ Alleles } & \multirow{2}{*}{ ST } \\
\hline & $\operatorname{rec} A$ & gapA & guaA & atpD & nuoD & $p p s A$ & mutM & \\
\hline $56 / 2012$ & 74 & $157^{a}$ & $273^{a}$ & $130^{a}$ & 139 & $186^{a}$ & $148^{a}$ & $498^{b}$ \\
\hline $57 / 2012$ & $150^{a}$ & 18 & $274^{\mathrm{a}}$ & 6 & $142^{\mathrm{a}}$ & $185^{\mathrm{a}}$ & $148^{\mathrm{a}}$ & $499^{b}$ \\
\hline $62 / 2012$ & 74 & $157^{\mathrm{a}}$ & $273^{a}$ & $130^{a}$ & 139 & $186^{a}$ & $148^{\mathrm{a}}$ & $498^{b}$ \\
\hline
\end{tabular}

${ }^{a}$ New alleles. ${ }^{b}$ New STs.

\section{Discussion}

An increase of nosocomial infections caused by S. maltophilia strains has been observed all over the world in recent years. According to Chang et al. [27], the prevalence of S. maltophilia infections in the global population increased from $1.3 \%$ to $1.7 \%$ between 2007 and 2012. The isolation of these bacteria from blood samples as an etiological factor of bacteremia is of particular concern $[28,29]$. In this study we characterized 94 S. maltophilia isolates by phenotypic and genotypic methods, including 79 isolates from patients hospitalized in one tertiary Warsaw hospital, of which 27 were isolated from blood. S. maltophilia is an opportunistic pathogen characterized by intrinsic resistance to many different groups of antibiotics, including aminoglycosides and almost all $\beta$-lactams. Trimethoprimsulfamethoxazole is still primarily the drug of choice for the treatment of $S$. maltophilia infections [2,30]. Screening studies conducted under the SENTRY Antimicrobial Surveillance Program, 2009-2012, showed resistance to trimethoprim-sulfamethoxazole among only $4 \%$ of $S$. maltophilia isolates from patients hospitalized with pneumonia in the United States and among $2 \%$ of isolates from European hospitals [31]. Likewise, all 94 isolates from the Warsaw hospital and from outpatients tested in this study turned out to be susceptible to trimethoprim-sulfamethoxazole. However, in the last 10 years, an increase in the number of isolates resistant to this chemotherapeutic agent has been observed in various regions of the world [28,32]. The second commonly used group of drugs in the treatment of infections caused by S. maltophilia strains are the fluoroquinolones, mainly levofloxacin $[28,33,34]$. Despite the high cure rates of $S$. maltophilia infections with levofloxacin monotherapy, a trend towards the selection of resistance to this fluoroquinolone has been observed [33]. Among the 94 S. maltophilia isolates tested in this study using the Etest with levofloxacin, $7 \%$ of the resistant isolates and $39 \%$ of the intermediate isolates were identified. Determination of the MIC value of antibiotics is a more accurate and reliable method of testing the drug susceptibility of bacteria than the disc-diffusion method. This is especially true specially in cases of obtaining results close to the cut-off points which determine the susceptibility 
of the isolate. However, some diagnostic laboratories issue results based solely on the disc-diffusion method.

The following antibiotics may also be used to treat S. maltophilia infections, mainly those caused by trimethoprim-sulfamethoxazole-resistant strains: minocycline, tigecycline, and the less frequently used ceftazidime and ticarcillin-clavulanate [28,35]. All 94 isolates tested in our study were susceptible to minocycline. Recently, the United States Food and Drug Administration (FDA) and the European Medicines Agency (EMA) approved a new drug, cefiderocol, which also presents high activity against $S$. maltophilia strains [36].

The main cause of difficulties in the treatment of infections caused by S. maltophilia is the intrinsic and acquired resistance of these strains to a wide range of antibiotics and chemotherapeutic agents. All strains produce L1 and L2 $\beta$-lactamases, which confer intrinsic resistance to $\beta$-lactam antibiotics [4]. The second important mechanism of resistance is active drug removal from bacterial cells by MDR efflux pumps. The sequencing of genomes of the S. maltophilia strains revealed the presence of MDR efflux systems from the RND family $(n=8)$, ABC family $(n=4)$, and MFS family $(n=1)[12-15,17]$. The SmeDEF and SmeABC efflux systems were detected in almost all studied strains [8,37]. However, no analysis of the occurrence of so many MDR efflux pumps in such a large group of isolates as in our study has been performed so far. Recent studies of the whole-genome sequencing of 375 strains isolated from different environments (from humans, animals, and the natural environment) focused mainly on the genogroup organization and diversity of S. maltophilia [38]. Moreover, mobile genetic elements and some antimicrobial-resistance genes were identified. However, no in-depth analysis of the occurrence of MDR efflux pumps was performed. Only the presence of genes encoding RND efflux pump proteins as subunit AcrA, and not the more closely identified permease subunit of the transporter, was signaled [38].

We demonstrated the prevalence of all MDR efflux systems from the RND $(n=8)$ and $\mathrm{ABC}(n=2)$ families among the majority of tested human isolates, including those derived from blood. The least-frequent MDR efflux pump in the studied isolates was SmeABC, which was detected in only 72 out of 94 isolates. It is known that contrary to MFS pumps, both RND and $\mathrm{ABC}$ efflux pumps have a wide substrate range, extruding trimethoprimsulfamethoxazole, fluoroquinolones, aminoglycosides, tetracyclines, $\beta$-lactams, and chloramphenicol $[4,8,37]$. Overexpression of these MDR efflux systems may result in resistance or decreased susceptibility in S. maltophilia strains. Thus, it could be the cause of therapeutic failure. Recently, it was noted that vitamin K3 induces the expression of the smeVWX efflux pump genes and confers resistance to quinolones, chloramphenicol, and tetracycline [39].

Generally, S. maltophilia isolates exhibit high genetic diversity. Genotyping of these isolates can be performed by various methods, such as PFGE, MLST, multilocus variable number of tandem repeat analysis (MLVA), restriction fragment length polymorphism analysis (RFLP) of the gyrB gene, amplified fragment length polymorphism analysis (AFLP) like repetitive extragenic palindromic-PCR (Rep-PCR), and enterobacterial repetitive intergenic consensus PCR (ERIC-PCR) $[18,19,24,26,40]$. The PFGE method is still considered the "gold standard" in the molecular typing of bacterial isolates, including S. maltophilia. PFGE analysis makes it possible to prove how closely related isolates tested in the laboratory are, and whether an outbreak of infections has occurred. However, unlike PFGE, methods based on PCR and sequencing allow for the comparison of results obtained in various laboratories around the world. Recently, bacterial whole-genome sequencing (WGS) has been used in scientific and epidemiological research [20].

Genotyping by PFGE and MLST is most commonly used to determine the relationship of $S$. maltophilia isolates worldwide $[18,19,24,26]$. In this study, the PFGE analysis revealed a clonal relationship with $100 \%$ similarity among two sub-PTs: the first included nine bloodstream isolates derived from patients of one hospital ward, and the second included six. It should be emphasized that the presence of genes encoding both the eight pumps from the RND family and the two pumps from the ABC family was found in all these isolates. The MLST genotyping of isolates representative of these two sub-PTs showed that they 
belong to the same new MLST profile, named as ST498. On the other hand, in PFGE, only 81.9\% similarity was observed between the two sub-PTs: A1 PT and A3 PT, despite these isolates belonging to the same MLST profile (ST498). Moreover, a correlation with 100\% similarity in PFGE analysis was observed for the other three pairs of isolates. The MLST analysis revealed that one of these isolates (classified under D1 PT) belonged to another new profile, named ST499. Both new ST profiles were deposited in public databases for molecular typing. Apart from this small group of above-mentioned 21 isolates, molecular genotyping revealed no other epidemiological incidents in both groups of hospitalized patients and outpatients. Recently, some data has been published showing the transmission of S. maltophilia strains not only between patients but also via items such as taps and beds in hospital wards [2,28,41]. PFGE and MLST genotyping were performed on 21 clinical isolates (from blood and urine) and 9 environmental isolates of $S$. maltophilia recovered from faucets in the emergency department in a Mexican tertiary care hospital. All nine environmental isolates showed $100 \%$ similarity to clinical blood isolates and were grouped into three PTs and two STs [28]. In our study, the bloodstream isolates with $100 \%$ similarity, grouped into two sub-PTs and the same ST498, were isolated over a long period of time (i.e., 3 years). Therefore, this may indicate the spread of $S$. maltophilia from the environment to patients. It is well known that strains of $S$. maltophilia are ubiquitous in the aquatic environment [1-3]. Perhaps, in this case, the S. maltophilia strains could live in the hospital ward environment (e.g., in taps or washbasin siphons), and sometimes be transmitted to immunocompromised patients, where the infection develops. The phenomenon of infecting patients with hospital strains living in the moist environment is not limited to Stenotrophomonas strains. This situation was described and epidemiologically investigated in the case of other bacteria species, such as Acinetobacter [42], Pseudomonas [43], and several Enterobacteriaceae species [44].

\section{Conclusions}

Although a large genetic diversity is generally observed among clinical isolates of S. maltophilia, we noticed two small outbreaks of infections in one of the hospitals in Warsaw over a three-year period. Most likely, the strains were being transferred from the hospital environment to immunocompromised patients. Moreover, two new MLST profiles (ST498 and ST499) were discovered and described among the studied S. maltophilia isolates. Analysis of the susceptibility profiles showed the possibility of using the first-line drug (i.e., trimethoprim-sulfamethoxazole) as well as minocycline in the treatment of infections. On the other hand, the reduction in susceptibility to levofloxacin demonstrated in $44 / 94$ isolates may indicate the ineffectiveness of using this fluoroquinolone in monotherapy. It should be emphasized that the presence of genes encoding eight efflux pumps from the RND family, as well as two efflux pumps from the ABC family, was shown in the majority of the 94 isolates. Such widespread occurrence of so many MDR efflux systems in clinical isolates of $S$. maltophilia indicates a high possibility of induction of the overexpression of these pumps, which would make these strains resistant to a wide spectrum of antibiotics.

Supplementary Materials: The following are available online at https:/ / www.mdpi.com/article/10. 3390/biology11010105/s1, Table S1: Presence of efflux pump genes in S. maltophilia isolates.

Author Contributions: Conceptualization, O.M.Z. and A.E.L.; methodology, O.M.Z. and A.E.L.; software, O.M.Z.; validation, O.M.Z., S.T. and A.E.L.; formal analysis, O.M.Z., S.T. and A.E.L.; investigation, O.M.Z. and A.E.L.; resources, O.M.Z.; data curation, O.M.Z. and A.E.L.; writingoriginal draft preparation, O.M.Z. and A.E.L.; writing—review and editing, O.M.Z., S.T. and A.E.L.; visualization, O.M.Z.; supervision, S.T. and A.E.L.; project administration, O.M.Z. and A.E.L.; funding acquisition, O.M.Z., S.T. and A.E.L. All authors have read and agreed to the published version of the manuscript.

Funding: This research was funded the grant from the National Science Center, Poland, no. 2016/21/ N/NZ7/03336. 
Institutional Review Board Statement: The clinical samples were part of the routine diagnostic procedure in microbiology laboratories and therefore the Institutional Review Board Statement was not applicable.

Informed Consent Statement: The clinical samples were part of the routine diagnostic procedure in microbiology laboratories and therefore the informed consent statement was not applicable.

Data Availability Statement: New sequence types were submitted to the S. maltophilia MLST database: ST498 (https:/ / pubmlst.org/bigsdb?page=profileInfo\&db=pubmlst_smaltophilia_seqd ef\&scheme_id=1\&profile_id=498, accessed on 19 June 2020) and ST499 (https:/ / pubmlst.org/bigsdb ?page=profileInfo\&db=pubmlst_smaltophilia_seqdef\&scheme_id=1\&profile_id=499, accessed on 19 June 2020).

Acknowledgments: Research was carried out with the use of the CePT infrastructure financed by the European Union through the European Regional Development Fund as part of the Operational Program "Innovative Economy" for 2007-2013.

Conflicts of Interest: The authors declare no conflict of interest.

\section{References}

1. Brooke, J.S. Advances in the microbiology of Stenotrophomonas maltophilia. Clin. Microbiol. Rev. 2021, 34, e0003019. [CrossRef]

2. Brooke, J.S. Stenotrophomonas maltophilia: An emerging global opportunistic pathogen. Clin. Microbiol. Rev. 2012, $25,2-41$. [CrossRef]

3. Paez, J.I.; Costa, S.F. Risk factors associated with mortality of infections caused by Stenotrophomonas maltophilia: A systematic review. J. Hosp. Infect. 2008, 70, 101-108. [CrossRef] [PubMed]

4. Sánchez, M.B. Antibiotic resistance in the opportunistic pathogen Stenotrophomonas maltophilia. Front. Microbiol. $2015,6,658$. [CrossRef] [PubMed]

5. Alonso, A.; Martinez, J.L. Cloning and characterization of SmeDEF, a novel multidrug efflux pump from Stenotrophomonas maltophilia. Antimicrob. Agents Chemother. 2000, 44, 3079-3086. [CrossRef]

6. Zhang, L.; Xian-Zhi, L.; Poole, K. SmeDEF multidrug efflux pump contributes to intrinsic multidrug resistance in Stenotrophomonas maltophilia. Antimicrob. Agents Chemother. 2001, 45, 3497-3503. [CrossRef]

7. Garcia-Leon, G.; Hernandez, A.; Hernando-Amado, S.; Alavi, P.; Berg, G.; Martinez, J.L. A function of SmeDEF, the major quinolone resistance determinant of Stenotrophomonas maltophilia, is the colonization of plant roots. Appl. Environ. Microbiol. 2014, 80, 4559-4565. [CrossRef] [PubMed]

8. Chang, L.L.; Chen, H.F.; Chang, C.Y.; Lee, T.M.; Wu, W.J. Contribution of integrons, and SmeABC and SmeDEF efflux pumps to multidrug resistance in clinical isolates of Stenotrophomonas maltophilia. J. Antimicrob. Chemother. 2004, 53, 518-521. [CrossRef]

9. Gould, V.C.; Okazaki, A.; Avison, M.B. Coordinate hyperproduction of SmeZ and SmeJK efflux pumps extends drug resistance in Stenotophomonas maltophilia. Antimicrob. Agents Chemother. 2013, 57, 655-657. [CrossRef] [PubMed]

10. Chen, C.H.; Huang, C.C.; Chung, T.C.; Hu, R.M.; Huang, Y.W.; Yang, T.C. Contribution of resistance-nodulation-division efflux pump operon smeU1-V-W-U2-X to multidrug resistance of Stenotophomonas maltophilia. Antimicrob. Agents Chemother. 2011, 55, 5826-5833. [CrossRef]

11. Lin, C.W.; Huang, Y.W.; Hu, R.M.; Yang, T.C. SmeOP-tolCsm efflux pump contributes to the multidrug resistance of Stenotrophomonas maltophilia. Antimicrob. Agents Chemother. 2014, 58, 2405-2408. [CrossRef]

12. Crossman, L.C.; Gould, V.C.; Dow, J.M.; Vernikos, G.S.; Okazaki, A.; Sebaihia, M.; Saunders, D.; Arrowsmith, C.; Carver, T.; Peters, N.; et al. The complete genome, comparative and functional analysis of Stenotrophomonas maltophilia reveals an organism heavily shielded by drug resistance determinants. Genome Biol. 2008, 9, R74. [CrossRef] [PubMed]

13. Al-Hamad, A.; Upton, M.; Burnie, J. Molecular cloning and characterization of SmrA, a novel ABC multidrug efflux pump from Stenotrophomonas maltophilia. J. Antimicrob. Chemother. 2009, 64, 731-734. [CrossRef] [PubMed]

14. Lin, Y.T.; Huang, Y.W.; Liou, R.S.; Chang, Y.C.; Yang, T.C. MacABCsm, an ABC-type tripartite efflux pump of Stenotrophomonas maltophilia involved in drug resistance, oxidative and envelope stress tolerances and biofilm formation. J. Antimicrob. Chemother. 2014, 69, 3221-3226. [CrossRef]

15. Huang, Y.W.; Hu, R.M.; Chu, F.Y.; Lin, H.R.; Yang, T.C. Characterization of a major facilitator superfamily (MFS) tripartite efflux pump EmrCABsm from Stenotrophomonas maltophilia. J. Antimicrob. Chemother. 2013, 68, 2498-2505. [CrossRef]

16. Hu, R.M.; Liao, S.T.; Huang, C.C.; Huang, Y.W.; Yang, T.C. An inducible fusaric acid tripartite efflux pump contributes to the fusaric acid resistance in Stenotrophomonas maltophilia. PLoS ONE 2012, 7, e51053. [CrossRef] [PubMed]

17. Dulyayangkul, P.; Calvopiña, K.; Kate, J.; Heesom, K.J.; Avison, M.B. Novel mechanisms of efflux-mediated levofloxacin resistance and reduced amikacin susceptibility in Stenotrophomonas maltophilia. Antimicrob. Agents Chemother. 2021, 65, e01284-20. [CrossRef]

18. Gherardi, G.; Creti, R.; Pompilio, A.; Di Bonaventura, G. An overview of various typing methods for clinical epidemiology of the emerging pathogen Stenothophomonas maltophilia. Diagn. Microbiol. Infect. Dis. 2015, 81, 219-226. [CrossRef] 
19. Pompilio, A.; Crocetta, V.; Ghosh, D.; Chakrabarti, M.; Gherardi, G.; Vitali, L.A.; Fiscarelli, E.; Di Bonaventura, G. Stenotrophomonas maltophilia phenotypic and genotypic diversity during a 10-year colonization in the lungs of a cystic fibrosis patient. Front. Microbiol. 2016, 7, 1551. [CrossRef]

20. Rizek, C.F.; Jonas, D.; Paez, J.I.G.; Rosa, J.F.; Neto, L.V.P.; Martins, R.R.; Moreno, L.Z.; Rossi, A., Jr.; Levin, A.S.; Costa, S.F. Multidrug-resistant Stenotrophomonas maltophilia: Description of new MLST profiles and resistance and virulence genes using whole-genome sequencing. J. Glob. Antimicrob. Resist. 2018, 15, 212-214. [CrossRef]

21. Clinical and Laboratory Standards Institute (CLSI). M02: Performance Standards for Antimicrobial Disk Susceptibility Tests, 13th ed.; CLSI: Wayne, PA, USA, 2018.

22. Liofilchem Inc. Liofilchem MTS-MIC Test Strip Guide. 2020. Available online: https:/ /www.liofilchem.com/solutions/clinical/ $\mathrm{arm} / \mathrm{mts}$-mic-test-strip.html (accessed on 18 September 2021).

23. Clinical and Laboratory Standards Institute (CLSI). M100: Performance Standards for Antimicrobial Susceptibility Testing, 31st ed.; CLSI: Wayne, PA, USA, 2021.

24. Jumaa, P.A.; Sonnevend, A.; Pàl, T.; El Hag, M.; Amith, R.; Trad, O. The molecular epidemiology of Stenotrophomonas maltophilia bacteraemia in a tertiary referral hospital in the United Arab Emirates 2000-2004. Ann. Clin. Microb. Antimicrob. $2006,5,32$. [CrossRef]

25. Tenover, F.C.; Arbeit, R.D.; Goering, R.V.; Mickelsen, P.A.; Murray, B.E.; Persing, D.H.; Swaminathan, B. Interpreting chromosomal DNA restriction patterns produced by pulsed-field gel electrophoresis: Criteria for bacterial strain typing. J. Clin. Microbiol. 1995, 33, 2233-2239. [CrossRef]

26. Kaiser, S.; Biehler, K.; Jonas, D. A Stenotrophomonas maltophilia multilocus sequence typing scheme for inferring population structure. J. Bacteriol. 2009, 191, 2934-2943. [CrossRef]

27. Chang, Y.T.; Lin, C.Y.; Chen, Y.H.; Hsueh, P.R. Update of infections caused by Stenotrophomonas maltophilia with particular attention to resistance mechanisms and therapeutic options. Front. Microbiol. 2015, 6, 893. [CrossRef]

28. Cruz-Cordova, A.; Mancilla-Rojano, J.; Luna-Pineda, V.M.; Escalona-Venegas, G.; Cázares-Domínguez, V.; Ormsby, C.; FrancoHernández, I.; Zavala-Vega, S.; Andrés-Hernández, M.; Medina-Pelcastre, M.; et al. Molecular epidemiology, antibiotic resistance, and virulence traits of Stenotrophomonas maltophilia strains associated with an outbreak in a Mexican tertiary care hospital. Front. Cell. Infect. Microbiol. 2020, 10, 50. [CrossRef]

29. Insuwanno, W.; Kiratisin, P.; Jitmuang, A. Stenotrophomonas maltophilia infections: Clinical characteristics and factors associated with mortality of hospitalized patients. Infect. Drug Resist. 2020, 3, 1559-1566. [CrossRef]

30. Anđelković, M.V.; Janković, S.M.; Kostić, M.J.; Živković Zarić, R.S.; Opančina, V.D.; Živić, M.Ž.; Milosavljević, M.J.; Pejčić, A.V. Antimicrobial treatment of Stenotrophomonas maltophilia invasive infections: Systematic review. J. Chemother. 2019, 31, $297-306$.

31. Sader, H.S.; Farrell, D.J.; Flamm, R.K.; Jones, R.N. Antimicrobial susceptibility of Gram-negative organisms isolated from patients hospitalized with pneumonia in US and European hospitals: Results from the SENTRY Antimicrobial Surveillance Program, 2009-2012. Int. J. Antimicrob. Agents 2014, 43, 328-334. [CrossRef]

32. Wang, C.-H.; Lin, J.-C.; Lin, H.-A.; Chang, F.-Y.; Wang, N.-C.; Chiu, S.-K.; Lin, T.-Y.; Yang, Y.-S.; Kan, L.-P.; Yang, C.-H.; et al. Comparisons between patients with trimethoprim-sulfamethoxazole-susceptible and trimethoprim-sulfamethoxazole-resistant Stenotrophomonas maltophilia monomicrobial bacteraemia: A 10 year retrospective study. J. Microbiol. Immunol. Infect. 2016, 49, 378-386. [CrossRef]

33. Nys, C.; Cherabuddi, K.; Venugopalan, V.; Klinker, K.P. Clinical and microbiologic outcomes in patients with monomicrobial Stenotrophomonas maltophilia infections. Antimicrob. Agents Chemother. 2019, 63, e00788-19. [CrossRef]

34. Azimi, A.; Rezaei, F.; Yaseri, M.; Jafari, S.; Rahbar, M.; Douraghi, M. Emergence of fuoroquinolone resistance and possible mechanisms in clinical isolates of Stenotrophomonas maltophilia from Iran. Sci. Rep. 2021, 11, 9582. [CrossRef]

35. Hotta, G.; Matsumura, Y.; Kato, K.; Nakano, S.; Yunoki, T.; Yamamoto, M.; Nagao, M.; Ito, Y.; Takakura, S.; Ichiyama, S. Risk factors and outcomes of Stenotrophomonas maltophilia bacteraemia: A comparison with bacteraemia caused by Pseudomonas aeruginosa and Acinetobacter species. PLoS ONE 2014, 9, e112208. [CrossRef]

36. Nakamura, R.; Oota, M.; Matsumoto, S.; Sato, T.; Yamano, Y. In Vitro activity and In Vivo efficacy of cefiderocol against Stenotrophomonas maltophilia. Antimicrob. Agents Chemother. 2021, 65, e01436-20. [CrossRef]

37. Cho, H.H.; Sung, J.Y.; Kwon, K.C.; Koo, S.H. Expression of Sme efflux pumps and multilocus sequence typing in clinical isolates of Stenotrophomonas maltophilia. Ann. Lab. Med. 2012, 32, 38-43. [CrossRef]

38. Mercier-Darty, M.; Royer, G.; Lamy, B.; Charron, C.; Lemenand, O.; Gomart, C.; Fourreau, F.; Madec, J.-Y.; Jumas-Bilak, E.; Decousser, J.-W.; et al. Comparative whole-genome phylogeny of animal, environmental, and human strains confirms the genogroup organization and diversity of the Stenotrophomonas maltophilia complex. Appl. Environ. Microbiol. 2020, 86, e02919-19. [CrossRef]

39. Blanco, P.; Corona, F.; Sánchez, M.B.; Martínez, J.L. Vitamin K3 induces the expression of the Stenotrophomonas maltophilia SmeVWX multidrug efflux pump. Antimicrob. Agents Chemother. 2017, 61, e02453-16. [CrossRef]

40. Roscetto, E.; Rocco, F.; Carlomagno, M.S.; Casalino, M.; Colonna, B.; Zarrilli, R.; Di Nocera, P.P. PCR-based rapid genotyping of Stenotrophomonas maltophilia isolates. BMC Microbiol. 2008, 8, 202. [CrossRef]

41. Kardan-Yamchi, J.; Hajihasani, A.; Talebi, M.; Khodaparast, S.; Azimi, A.; Rahbar, M.; Fallah, F.; Douraghi, M. Intra-hospital dissemination of clinical and environmental isolates of Stenotrophomonas maltophilia from Tehran. Lett. Appl. Microbiol. 2021, 72, 325-331. [CrossRef] 
42. Nasr, P. Genetics, epidemiology, and clinical manifestations of multidrug-resistant Acinetobacter baumannii. J. Hosp. Infect. 2020, 104, 4-11. [CrossRef]

43. Pirzadian, J.; Harteveld, S.P.; Ramdutt, S.N.; van Wamel, W.J.B.; Klaassen, C.H.W.; Vos, M.C.; Severin, J.A. Novel use of culturomics to identify the microbiota in hospital sink drains with and without persistent VIM-positive Pseudomonas aeruginosa. Sci. Rep. 2020, 10, 17052. [CrossRef]

44. Kac, G.; Podglajen, I.; Vaupré, S.; Colardelle, N.; Buu-Hof, A.; Gutmann, L. Molecular epidemiology of extended-spectrum beta-lactamase-producing Enterobacteriaceae isolated from environmental and clinical specimens in a cardiac surgery intensive care unit. Infect. Control Hosp. Epidemiol. 2004, 25, 852-855. [CrossRef] 